\title{
Lattice Boltzmann simulation for flux change in laminar flow under oscillating boundary condition
}

\author{
Ryuta Ueda ${ }^{1}{ }^{*}$, Hitoshi Mikada ${ }^{1}$, Tada-nori Goto ${ }^{1}$, Junichi Takekawa ${ }^{1}$ \\ ${ }^{1}$ Dept. of Civil and Earth Res. Eng., Kyoto University
}

\begin{abstract}
The amount of oil production in the world is decreasing recently and it is of importance to seek the technological development for enhanced oil recovery (EOR) in place in the subsurface. Seismic stimulation is known as one of the methods of EOR. Recently, many laboratory experiments and field tests have been performed such as water, gas, chemical, or thermal injections to attempt the enhancement of oil production. Numerous observations show that seismic stimulation of oil reservoir may alter water and oil production. But the detailed mechanism of seismic stimulation is not fully understood. We attempt to understand the mechanism of the flux change in viscous laminar flow under oscillating boundary condition to simulate seismic EOR. We focus on flux change by the oscillated boundary wall because of partial high pressure gradient and flow velocity difference. In this study, we analyze a single-phase flow in various pore shapes and scales with two dimensional (2D) Lattice Boltzmann method (LBM). The results show pore scales and shapes are largely related with the flux change, because of the reduction of pressure loss and the flow velocity difference between the wall and flow.
\end{abstract}

\section{Introduction}

The amount of oil production in the world is decreasing recently and it is of importance to seek the technological development for enhancing the recovery of oil in place in the subsurface : Enhanced oil recovery(EOR). EOR can be achieved by several methods: water injection, gas injection, chemical injection, and thermal injection. Seismic stimulation is known as one of the methods of EOR. Many observations show that seismic stimulation of oil reservoir may alter water and oil production, because of altering the apparent viscosity coefficient, or contributing to the coalescence or dispersion of oil droplets ${ }^{1}$ ). Clamen and $\operatorname{Minton}(1972)^{2}$ ) showed flow velocity under moving pipes harmonically was lower than one under non-moving case. Pride et al. $(2008)^{3}$ ) attempt to simulate the mechanism of coalescence or dispersion of oil droplets with Lattice Boltzmann method (LBM). Beresnev ${ }^{4}{ }^{5}$ ) (2006 and 2009) attempted to analyze the phenomenon of oil droplet forced by seismic stimulation at the pore-throat. Zaaem et al.(2009) attempted to grasp the flux change of non-Newtonian fluid under vibration. But the detailed mechanism of seismic stimulation is not fully understood.

To use seismic EOR efficiently, we need to understand the mechanisms of macroscopic phenomenon generated by seismic stimulation: flux increment, pore-water pressure increment and relative permeability improvement. In this study, we attempt to demonstrate the flux change in viscous laminar flow under oscillating boundary condition for the simulation of interstitial flow. Here, we analyze a single-phase flow in various pore scales and shapes with 2D LBM scheme to discuss the possibility of flux change by pore shapes and scales.

\section{Method}

In this study, we analyze 2D incompressible flow. In incompressible viscous flow, the governing equation is Navier-Stokes equation (2a) and Mass equation $(2 b)$,

$$
\begin{gathered}
\rho \frac{\partial u_{i}}{\partial t}+\rho u_{j} \frac{\partial u_{i}}{\partial x_{j}}=-\frac{\partial p}{\partial x_{i}}+\mu \frac{\partial^{2} u_{i}}{\partial x_{j}^{2}} \\
\frac{\partial u_{i}}{\partial x_{i}}=0
\end{gathered}
$$

where $\rho$ is the fluid density, $u$ is the fluid velocity, and $\mathrm{p}$ is the fluid pressure.

CFD has some methods like FDM, FEM and particle methods. In this study, we use Lattice Boltzmann method (LBM). We use 2-dimensional 9-velocity $(2 \mathrm{~d} 9 \mathrm{v})$ model to simulate 2-dimensional incompressible viscous flow(figure.1) The square lattice LBGK model is expressed as:

$$
f_{i}^{n+1}=f_{i}^{n}-\frac{1}{\tau_{k}}\left(f_{i}^{n}-f_{i}^{n, e q}\right)
$$


where second term of the right side is collision term. $f_{i}^{n, e q}$ is the equilibrium distribution function, $\tau_{k}$ is the relaxation coefficient which controls the rate of approach to equilibrium. The density per node, $\rho$ and the macroscopic flow velocity, u $=(\mathrm{Ux}, \mathrm{Uy})$, are defined in the terms of the particle distribution function by

$$
\sum_{i=0}^{8} f_{i}=\rho, \quad \sum_{i=0}^{8} f_{i} \cdot c_{i}=\rho \boldsymbol{u}
$$

The equilibrium distribution functions $f_{i}^{n, e q}$ depend only on local density and velocity and they cau be chosen in the following form.

$$
f_{i}^{n, e q}=\rho\left(1+3 \boldsymbol{c}_{\boldsymbol{i}} \cdot \boldsymbol{u}+\frac{9}{2}\left(\boldsymbol{c}_{\boldsymbol{i}} \cdot \boldsymbol{u}\right)^{2}+\frac{3}{2} u^{2}\right)
$$

$\tau_{k}$ is relaxation coefficient, and this is defined in the terms of kinematic viscosity coefficient, time step, and divider width.

$$
\tau_{k}=3 v \frac{d x}{(d t)^{2}}+\frac{1}{2}
$$

We assume the solid is rigid. Considering the relative velocity between solid and liquid, we calculate the velocity of wall is zero. On the other hand, we add the internal force by oscillating boundary condition. In our simulation, we adopt the boundary conditions of Zou and $\mathrm{He}(1997)$.

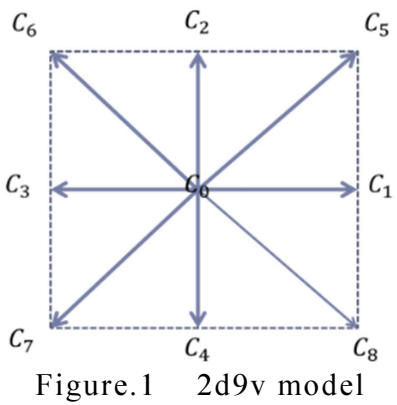

\section{Model}

Pore throats in rock have complex shape. Choi and Clayton $\left.(2000)^{6}\right)$, Quadrio and Sibilla $(2000)^{7}$ ', and Bewley $(2001)^{8}$ ) showed span wise or stream wise oscillation reduce the drag reduction and improve flow. So, we attempt to simulate the flow under various pore shapes to grasp the influence of the pore shapes (Figure.2).

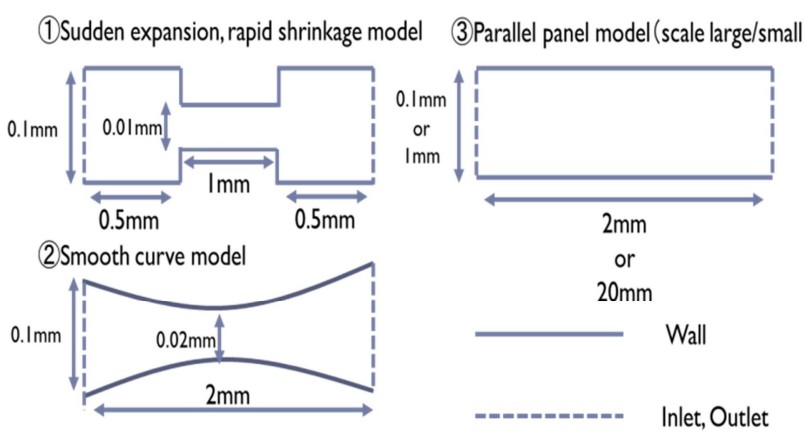

Figure.2 Simulation shape、(3)is two model: large and small.

In this simulation, we simulate two different oscillation: span wise and stream wise (Figure.3).

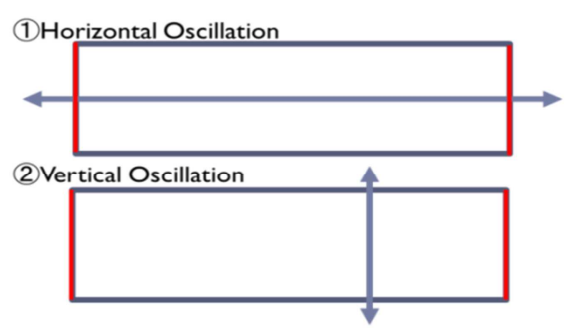

Fig.3 Oscillating Direction

\section{Result and Discussion}

In this study, we discuss two characteristics: amplitude and frequency. In frequency characteristic, a vertical axis is flux ratio: this is the proportion of the flux under oscillating bounrary to that under not oscillating, and a horizontal axis is frequency $(\mathrm{Hz})$. In amplitude characteristics, a vertical axis is also flux ratio, and a horizontal axis is amplitude $(\mathrm{m})$.

\section{(1) Stream wise (Horizontal) oscillation}

First, we discuss the possibility of flux change in parallel panel model. In model (3) large model, we generate the pressure gradient, $0.98(\mathrm{~Pa} / \mathrm{m})$. These figures (Figure.4, 5) show that oscillating wall in horizontal direction may cause the flux reduction.
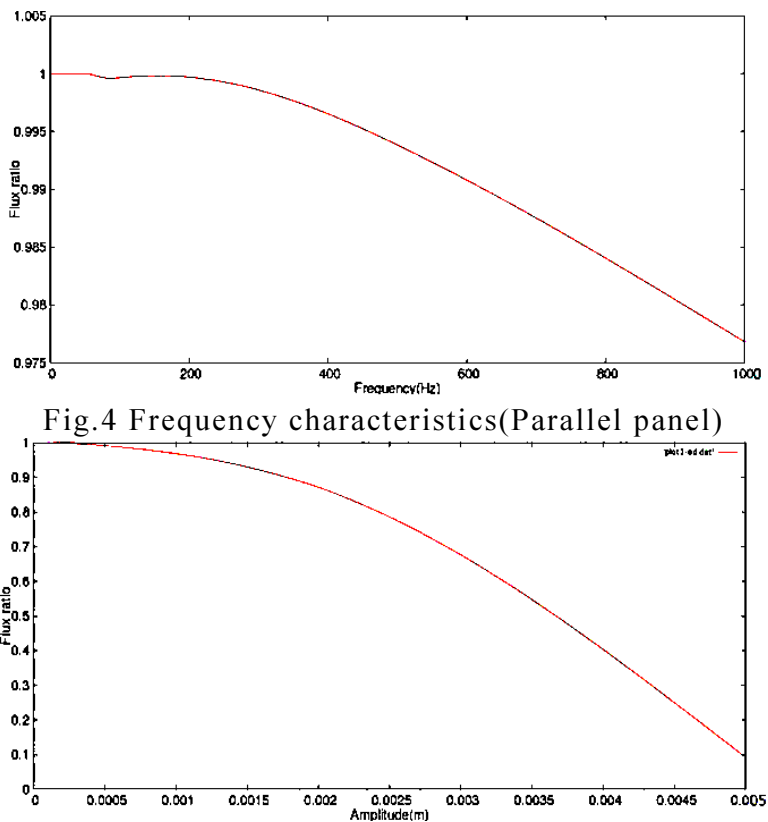

Fig.5 Amplitude characteristic (Parallel panel)

Second, we discuss the possibility of flux change in smooth curve model. In model (2), this figure shows that oscillating wall in horizontal direction may cause the flux increment. Especially, the oscillation of high frequency may cause the flux increment.

Third, we discuss the possibility of flux change in sudden expansion and rapid shrinkage model. In 


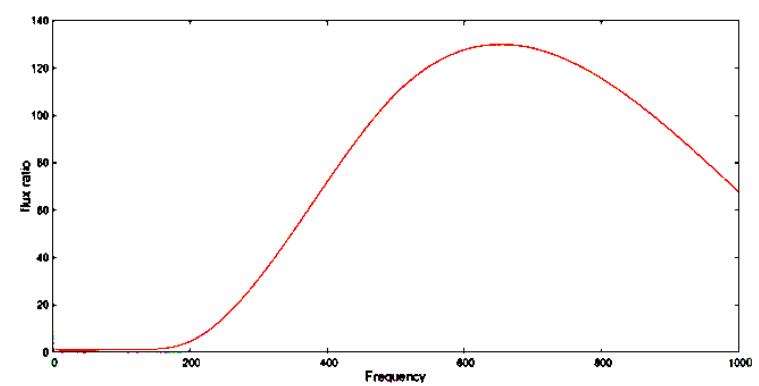

Fig. 6 Frequency characteristic(Smooth curve model) model (1), this figure shows that oscillation wall in horizontal direction may cause very large flux increment.

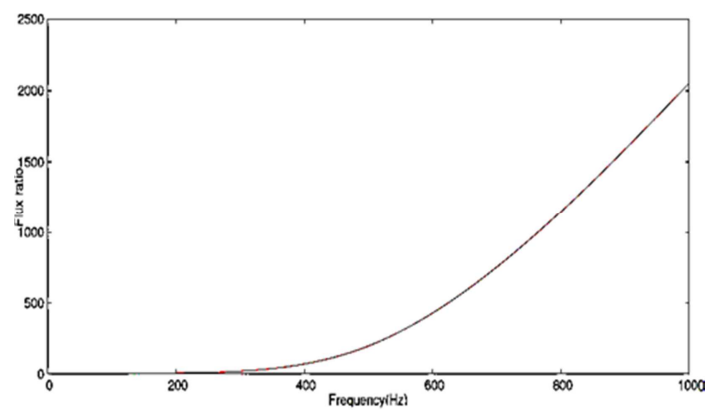

Fig. 7 Frequency characteristic

(Rapid expansion, rapid shrinkage model)

Finally, we discuss the flux change in small scale model. In model (3) small model, this figure shows that the oscillation in small pore throat cause smaller flux increment than that in large.

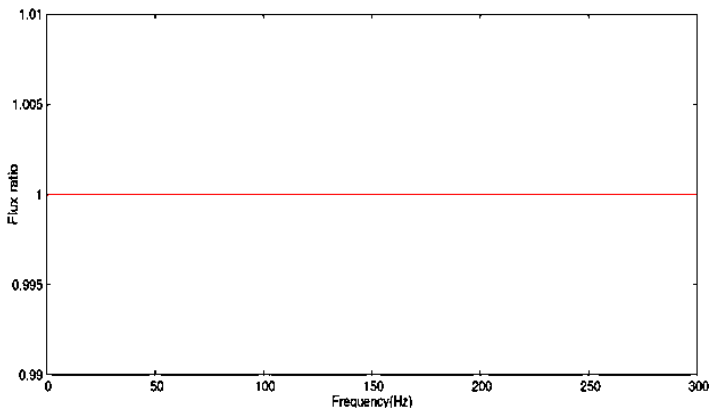

Fig.8 Frequency characteristic (Scale change)

\section{(2) Spanwise (Vertical) oscillation}

First, we discuss the possibility of flux change in parallel panel model. In model (3) large model, we generate the pressure gradient, $0.98(\mathrm{~Pa} / \mathrm{m})$. These figures (Figure.9, 10) show that oscillating wall in vertical direction may not cause flux change. So, in very low Reynolds flow, vertical oscillation is not effective to improve flow.

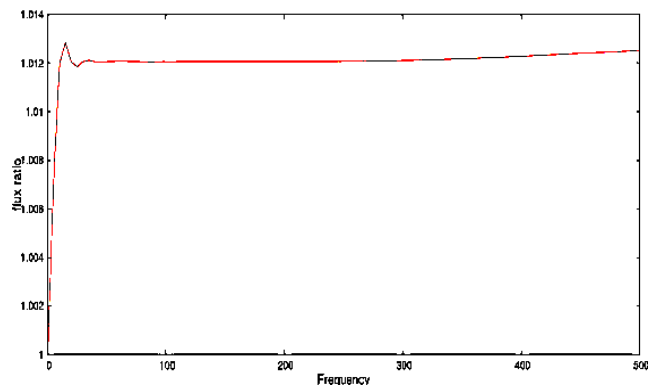

Fig.9 Frequency characteristic (Parallel panel)

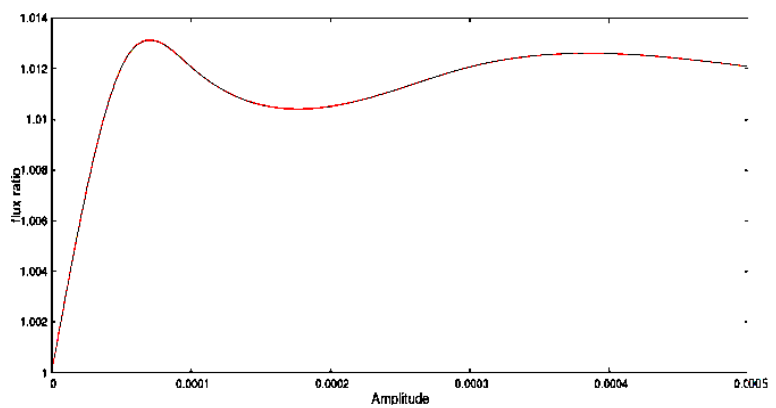

Fig.10 Amplitude characteristic (Parallel panel)

Second, we discuss the possibility of flux change in smooth curve model. In model (2), this figure shows that oscillating wall in vertical direction may cause a little flux increment under high frequency oscillation.

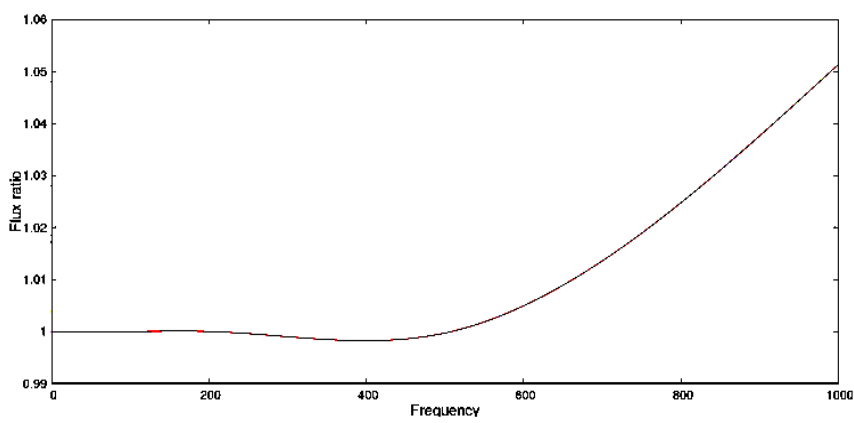

Fig. 11 Frequency characteristic(Smooth curve model) Third, we discuss the possibility of flux change in sudden expansion and rapid shrinkage model. In model (1), this figure shows that oscillation wall in vertical direction may cause a few percent flux increment under high frequency oscillation.

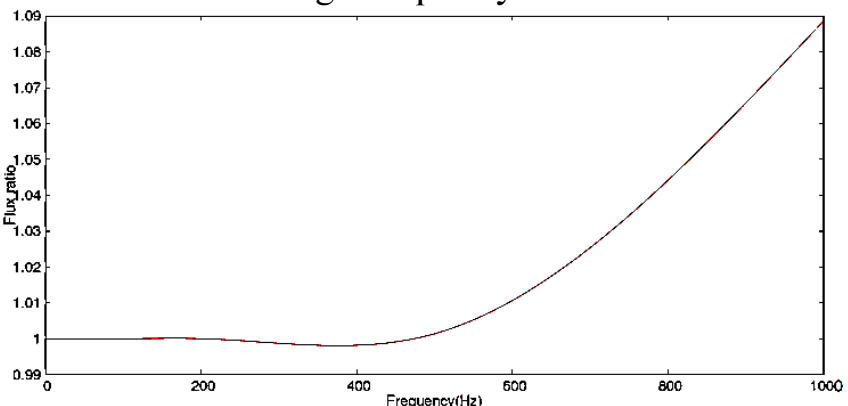

Fig. 12 Frequency characteristic

(Rapid expansion and rapid shrinkage model)

Finally, we discuss the flux change in small scale model. In model (3) small model, this figure shows that the oscillation in small pore throat cause smaller flux increment than that in large.

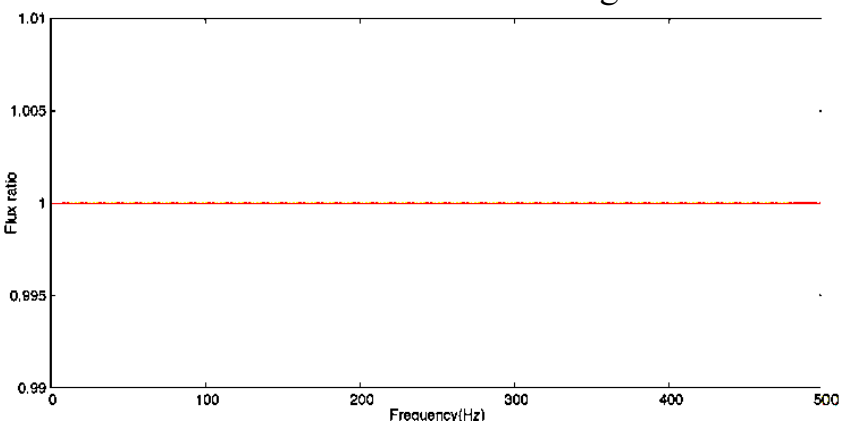

Fig. 13 Frequency characteristic (Small scale)

(3)The assumption of flux change We assume three reasons to change the flux: 
increment or reduction.

First, by large streamwise oscillation, large velocity difference between the wall and the flow central is generated. In low Reynolds flow, the horizontal wall oscillations propagate in fluid instantly. But if the delay of propagation is generated, the flow resistance is higher than not generated. So, the central velocity of flow under oscillating boundary is lower than under not oscillating.

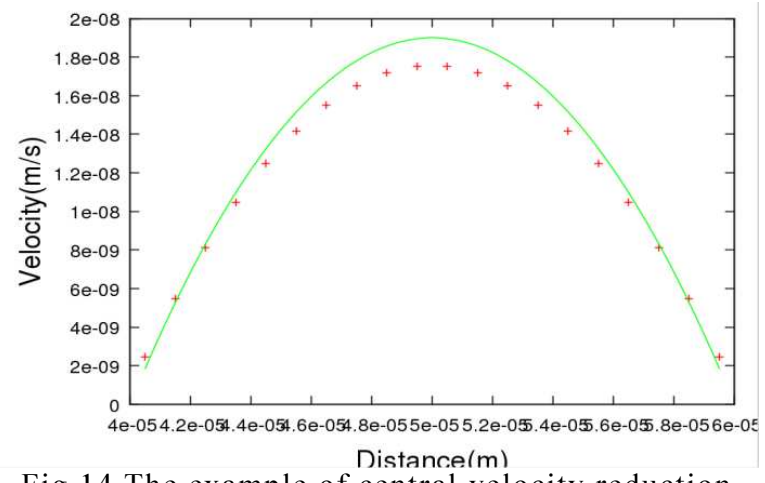

Fig. 14 The example of central velocity reduction (Sudden expansion and rapid shrinkage model)

Second, by large spanwise oscillation, partial pressure gradient is generated. The vertical velocity difference between the flow and the wall cause the concentration/dispersion of fluid. If the oscillation generate the concentration of fluid, the fluid flow out in stream direction.

Finally, oscillating boundary causes improving pressure loss. Choi and Clayton $\left.(2000)^{6}\right)$, Quadrio and Sibilla $(2000)^{7)}$, and Bewley $\left.(2001)^{8}\right)^{2}$ suggest that wall oscillation cause the possibility of flux increment, because of drag reduction and pressure loss. In this simulation, sudden expansion, rapid shrinkage zone and small pore throat generate pressure loss (Figure.14). So, in this model, oscillation causes the possibility of large flux increment.

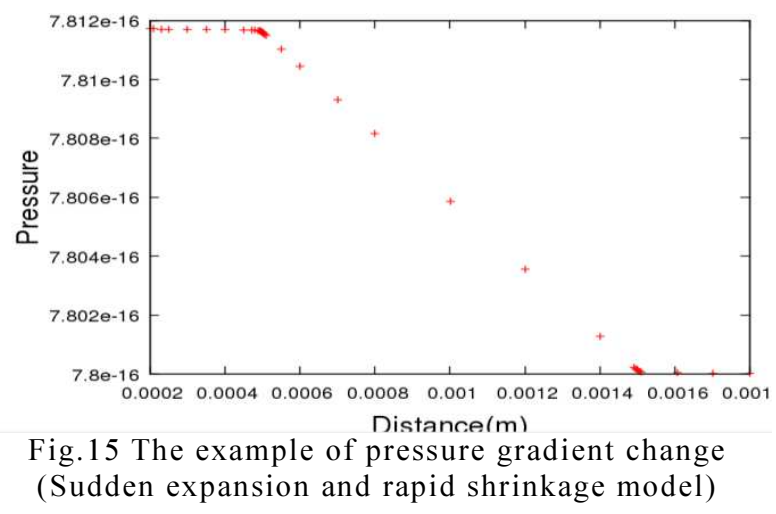

\section{Conclusion and future work}

In this study, we discuss the possibility of flux change by pore scales or shapes under oscillating boundary condition with LBM.

In consequence, the zigzagging models, especially sudden expansion and rapid shrinkage model, generate larger flux change than parallel panel model.

For next stage, we try to evaluate following three points. First, we attempt to consider two-phase flow. In two-phase flow, the oscillating boundary conditions generate the reduction of interfacial tension, improvement of relative permeability and the coalescence or dispersion of oil droplets. The consideration of the combination of fluid and solid to simulate flux change under $\mathrm{P}$ wave is also important due to volume change by $\mathrm{P}$ wave. Finally, we attempt to simulate realistic pore throat model like Gaussian Random Field model.

\section{REFERENCES}

1) Beresnev, I.A. and Paul A.J. (1994) Elastic-wave stimulation of oil production: A review and results, Los Alamos National Laboratory, Geophysics, 59, 6, 1000-1017.

2) Clamen, M. and Minton, P., (1976) An experimental investigation of flow in an oscillating, J. Fluid Mech., 81, 421-431.

3) Pride, S.R., Flekkoy, E. and Aursjo, O., (2008) Seismic stimulation for enhanced oil recovery, Geophysics, 73, O23-P35.

4) Beresnev, I.A., (2006) Theory of vibratory mobilization on nonwetting fluids entrapped in pore constrictions, Geophysics, 71, 6, N47-N56.

5) Beresnev, I.A, (2009) Viscosity effect in vibratory mobilization of residual oil, Geophysics, 75, 4, N79-N85

6) Zaaem, M.A., Lapin, S., Matveev, K., (2009) The Effect of Vibration on Flow Rate of Non-Newtonian Fluid, Proc. Fourth SIAM Conference on Mathematics for Industry, 137-141.

7) Kwing-So, C. and Brian, R. C., (2000) The mechanism of turbulent drag reduction with wall oscillation, International Journal of Heat and Fluid Flow, 22, 1-9

8) Maurizio, Q. and Stefano, S.(2000) Numerical simulation of turbulent flow in a pipe oscillating around its axis, J.Fluid Mech.,424, 217-241

9) Thomas, R. B., (2001) Flow control: new challenges for a new Renaissance, Progress in Aerospace Science, 37, 21-58 